\title{
Effects of physical and mental demands on shoulder muscle fatigue
}

\author{
Ranjana K. Mehta ${ }^{\mathrm{a}^{*}}$ and Michael J. Agnew ${ }^{\mathrm{b}}$ \\ ${ }^{a}$ Departments of Cognitive and Learning Sciences and Kinesiology and Integrative Physiology, Michigan \\ Technological University, 1400 Townsend Drive, Houghton, MI, USA \\ ${ }^{\mathrm{b}}$ Department of Industrial \& Systems Engineering, Virginia Tech, Blacksburg, VA, USA
}

\begin{abstract}
Mental demands have been associated with increased risk of injuries; however, its influence on muscle fatigability remains unclear. The aim of this study was to investigate the interaction of mental workload and physical workload on muscle fatigability during repetitive shoulder work. Twelve young participants, balanced by gender, performed shoulder abduction exercises until exhaustions at three levels of physical workload (low (5\% maximum voluntary contraction (MVC)), moderate $(35 \% \mathrm{MVC})$, and high $(55 \% \mathrm{MVC}))$ in the absence and presence of a mental arithmetic test. Endurance time and rate of strength decline were employed as indicators of muscle fatigue. Concurrent physical and mental processing was found to adversely decrease muscle endurance by $\sim 25 \%$ at the moderate intensity level. Furthermore, concurrent demands were associated with faster rate of strength decline compared to the control, irrespective of the physical intensity level. Findings from the current study provide evidence of the adverse effects of mental workload on muscle capacity (i.e., endurance and fatigue). It is therefore important to consider potential changes in worker capacity with concurrent physical and cognitive demands before designing work tasks/products.
\end{abstract}

Keywords: physical workload, mental workload, fatigue, endurance

\section{Introduction}

Occupational tasks place both physical and psychological strain on workers, which can ultimately result in the development of work-related musculoskeletal disorders (WMSDs). Yet, existing research focusing on WMSD development or ergonomic interventions do not address the interaction between mental and physical demands associated with occupational tasks. Much of the occupational injury research has focused on physical risk factors, such as forceful application, repetition, and overexertion [1]. A few studies have demonstrated adverse effects of psychological stressors, such as intensified workload and time pressure, on worker health and performance $[2,11]$. Although the intensity of these stressors alone may not be causal in the development of WMSDs, their interactions with physical demands have consistently been associated with increased muscle tension and cardiovascular responses $[5,9]$.

According to the National Research Council/Institute of Medicine [14] model of WMSD causation, overall workplace task demands (due to combined physical and mental demands) can affect physiological responses and fatigue (worker capacity) that may ultimately lead to the development of WMSDs. While independent causal relationships between the different types of demand (i.e., physical and mental) and physiological response (illustrated in Figure 1) have been demonstrated in experimental studies [3, 17], relatively little research, however, has investigated the influence of concurrent physical and mental demands (i.e., overall demands) on physiological measures. Some studies have reported increases in shoulder muscle activity due to mental demands placed on operators during computer work $[7,11,19]$.

*Corresponding author. Ranjana K. Mehta, Department of Cognitive and Learning Sciences/Department of Kinesiology and Integrative Physiology, Meese G08, Michigan Technological University, Houghton, MI 49931. Tel.: +1 906-487-1132; Fax: +1 906-487-1094; E-mail: ranjanam@mtu.edu 
The increased muscle activity is suggested to result from co-contraction of the musculature to stiffen a joint, in an attempt to provide additional stabilization [16]. However, over prolonged duration, increased muscle tension could also lead to the development of WMSDs [10].

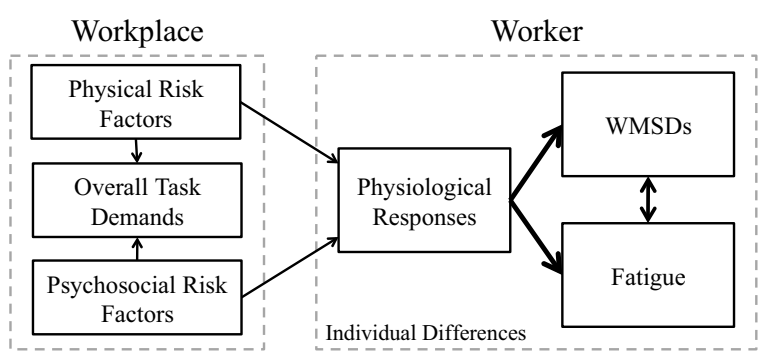

Figure 1. Conceptual model of WMSD development (Adapted from NRC/Institute of Medicine model of WMSD causation)

Despite increasing evidences of the detrimental effects of concurrent demands on physiological responses, existing research has not yet quantified their effects on muscle capacity. It could be argued that continued exposure to combined loads may increase muscular effort [9], which may ultimately influence muscle capacity (Figure 1). However, it remains unclear if cognitive stressors at work can negatively influence worker muscle capacity. Muscle capacity has previously been measured using muscle endurance (i.e., time to task failure) or decline in force generating capacity (i.e., strength decline). Ergonomic tools to determine work demands or work-rest schedules still rely on existing endurance and strength data that account for physical demands alone. Under conditions of physical and mental processing, overall demands can be underestimated and worker capacity may potentially be overestimated. Thus, it is imperative to determine changes in muscle capacity when workers perform concurrent physical and mental work, such that appropriate workload determination or guidelines are based on adjusted capacity and effective administrative controls are employed to further minimize the risk of injuries due to concurrent demands.

The purpose of this study was to investigate the influence of mental workload on muscle fatigability during repetitive shoulder work. It was hypothesized that mental workload will adversely affect muscle fatigue, and that these changes will be dependent on physical workload levels.

\section{Methods}

Upon IRB approval, twelve participants (balanced by gender) were recruited, whose mean (SD) age, stature, and body mass were 21.21 (1.75) years, 1.51 (0.49) $\mathrm{m}$, and 67.89 (11.08) kg, respectively. Only participants with no prior history of musculoskeletal injuries or pain were included in the study.

\subsection{Experimental design}

Independent variables manipulated in the study were physical workload, presented as percent maximum voluntary contraction (MVC) at low, moderate, and high levels $(15 \%, 35 \%, 55 \% \mathrm{MVC}$, respectively), and cognitive demand, presented as absence (control) and presence (concurrent demand) of a mental arithmetic task. The study was a $3 \times 2$ repeated measures design, with participants performing each treatment condition on separate experimental days, with an additional preliminary session at the beginning of the study. A balanced Latin Square design was employed to determine the order of the treatment conditions to avoid any order or learning effects.

\subsection{Procedures}

At the preliminary session, participants were familiarized with the experimental protocol and were provided with practice trials of the physical and mental task. To determine the levels of physical workload, participants performed a series of MVCs of the shoulder. The task required them to be seated in a dynamometer Biodex $^{\mathrm{TM}}$ System 3 Pro Medical System, Shirley, New York, USA), secured at the shoulder and waist, with their right shoulder abducted at $90^{\circ}$ (Figure 2). They were instructed to abduct their arm against the dynamometer arm with maximal strength. Three MVC trials were performed, and the maximum value from the three MVCs was used to determine physical workload levels.

At the start of each experimental session, participants performed a pre-MVC utilizing the same posture and setup as in the preliminary session (Figure 2). Upon adequate rest, they underwent the endurance test at one level of physical workload level. The endurance test required participants to perform intermittent static exertions, with $15 \mathrm{~s}$ of work and $15 \mathrm{~s}$ of rest (duty cycle of $50 \%$ ), until exhaustion or up to a limit of one hour. Visual feedback was presented as a series of square waves displaying work and rest periods, thereby visually controlling the duration of the 
work-rest cycle. In the control conditions, participants performed these exertions until exhaustion with no additional tasks. In the concurrent condition, participants performed these exertions while continuously performing a serial-three mental arithmetic task during the work periods. The task required participants to multiply a one- or two-digit number randomly presented at the start of each work period and they were instructed to perform as many multiplications as possible within the work period of 15 seconds as accurately as they could, while maintaining their force levels. Upon exhaustion (i.e., participants could no longer maintain their force at the required exertion level), participants performed a post-MVC, which marked the end of that experimental session.

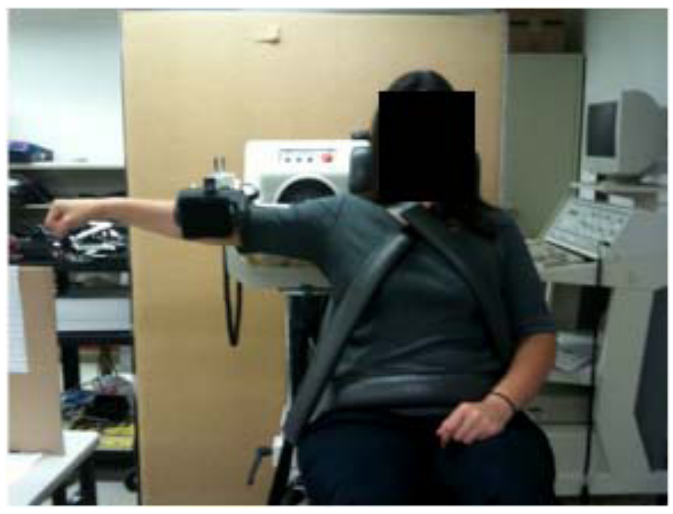

Figure 2. Participant posture for the endurance test

\subsection{Dependent measures}

The dependent measures included endurance time and rate of strength decline. Endurance time was defined as the time to exhaustion while performing exertions at the required target force levels. Rate of strength decline was determined as the percentage reduction in shoulder strength ((post-MVC - pre$\mathrm{MVC}$ )/ pre-MVC) relative to individual endurance time.

\subsection{Statistical analyses}

To evaluate the effect of physical and mental workload on muscle fatigue, a 3 (physical workload) x 2 (mental workload) measures analysis of variance (ANOVA) was conducted for both dependent measures. Where required, post-hoc comparisons were conducted using Tukey's Honestly Significant Difference (HSD) test. Statistical significance was de- termined when $p<0.05$. All statistical analyses were conducted using JMP 7.0 (SAS Institute Inc., Cary, $\mathrm{NC})$. Summary data is represented as mean (SD).

\section{Results}

\subsection{Endurance time}

In general, all participants could complete the endurance test (of one hour duration) at $15 \% \mathrm{MVC}$, both in the absence and presence of the mental arithmetic task. However, endurance time decreased significantly with increasing physical workload $(p<0.0001)$. More importantly, additional mental workload was found to decrease endurance time ( $p=$ 0.032). Furthermore, a significant interaction effect of physical and mental workload was found on endurance time $(p=0.011)$, with shorter endurance time observed only during moderate force levels (i.e. at $35 \% \mathrm{MVC})$ in presence of mental arithmetic (12.17 $\mathrm{min})$ compared to the control condition (12.17 (7.88) vs. 16.33 (11.83) minutes, respectively). Figure 3 illustrates this interaction effect.

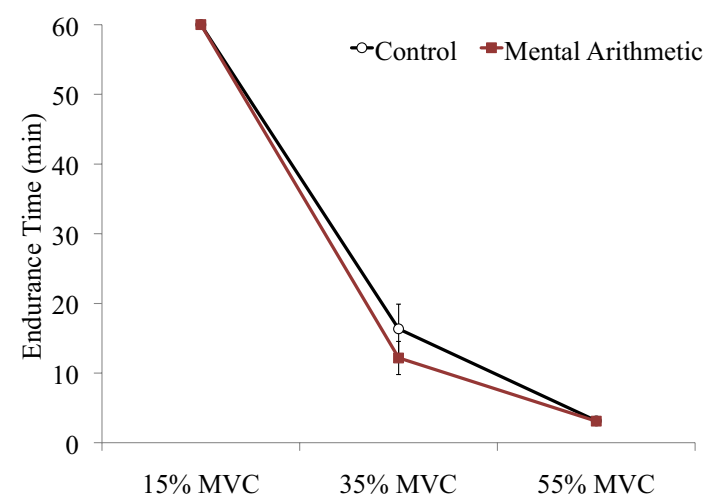

Figure 3. Interactive effect of physical and mental workload on endurance time. Error bars represent standard error.

\subsection{Rate of strength decline}

Main effects of physical $(p<0.0001)$ and mental workload $(p=0.02)$ were found on rates of strength decline (Figure 4). Greater strength decline was observed at higher physical workload levels and in conditions requiring concurrent physical and mental processing. 




Figure 4. Main effects of physical and mental workload on rate of strength decline. Error bars represent standard error.

\section{Discussion}

The aim of this study was to investigate whether concurrent physical and mental workload interact and influence muscle fatigue. As hypothesized, concurrent demands adversely affected muscle endurance and resulted in a faster rate of strength decline. These findings suggest that additional mental demands can decrease workers' capacity and may put them at a greater risk of injuries.

Shorter endurance times during concurrent demand conditions (compared to the control conditions) may be attributed to increased attentional resources allocated to the mental arithmetic task. It is argued that the lack of available attentional resources, which was needed to increase the drive to motor neurons to maintain the required force levels, was responsible for the decreased muscle capacity $[8,20]$. Similar findings have been reported by Yoon et al. [21], who demonstrated decreased endurance time due to cognitive stressors at low-level sustained contractions. Furthermore, greater decreases in rate of strength decline were observed during concurrent demand conditions compared to the control. A similar decrease in peak force generation during simultaneous cognitive processing was demonstrated by Macdonell $\&$ Keir [12]. Thus, it can be argued that mere distraction due to the mental task did not adversely affect endurance time. Rather, earlier onset of peripheral fatigue contributed to the decrease in muscle endurance, highlighting that mental workload can influence peripheral fatigue [6].

The levels of physical demand influenced the effect of mental demand on muscle endurance. Endur- ance time decreased by $\sim 25 \%$ in the presence of $\operatorname{cog}$ nitive demand at moderate force level (i.e., at 35\% MVC) compared to other levels (0-2\% decrease). Each participant could complete the endurance task at $15 \%$ MVC (i.e., they could perform the task for 60 minutes), irrespective of the mental demand condition. At 55\% MVC, participants could only perform the task for about 2-4 minutes. In comparison, at $35 \%$ MVC participants performed the arithmetic task for a longer duration, which may have induced greater levels of mental fatigue and thereby adversely affected motivation. Since central fatigue is governed by parameters such as motor fatigue and motivation [4] it is possible that participants may have reached a state of central fatigue at moderate force levels compared to other force levels.

Endurance time and rate of strength decline are the commonly used fatigue indicators [18, 21]. While both measures provide similar information about changes in capacity over a period of time, they may also be considered as indicators of worker performance and health. Results from this study highlight shorter endurance times with additional mental workload. This may be translated to decrease in worker performance (i.e., motor performance) with concurrent tasks. A decrease in performance may lead to increased errors (or accidents), and may in turn affect worker safety and/or morale. The study also demonstrated a faster rate of strength decline with concurrent tasks, which may adversely affect the worker gradually, putting him at a higher risk of injury.

Issues concerning manipulation of mental workload in the current research warrant some discussion. The scope of this research was to investigate the effects of mental workload and its interaction with physical workload on muscle fatigue. With the advancement of industrial automation, an increasing amount of mental workload is placed on workers along with the physical demands due to the tasks. As such, intensified workload has been strongly associated with pain symptoms and risk of WMSDs [1]. Numerous laboratory studies have employed cognitive stressors, such as the Stroop Color Word test [12, 13] and mental arithmetic tasks [3] to investigate the effects of mental workload and/or stress on physiological responses. In particular, mental arithmetic has been used widely to induce mental workload/stress as it has shown distinct changes in physiological responses [15] and requires cognitive processing that is representative of mental demands placed on workers at their daily jobs.

To alleviate adverse effects of concurrent demands on muscle fatigability and decreased worker capacity, 
several recommendations are provided. First, proactive workload determination of work tasks should be based on the overall demands of the task and modified capacity of the worker to minimize undue strain on the worker. Second, concurrent demands exacerbate muscle fatigability. Thus, additional rest breaks should be provided to hinder fatigue progression for occupational tasks that are characterized with high levels of physical and mental workload. Third, tasks should be redesigned such that the interference due to concurrent demands is minimized. This can be achieved by adopting different workload reduction strategies, such as serial versus parallel processing of physical and mental demand, employing design principles from human information processing domain (such as affordances and redundant coding) to reduce cognitive demands, and varying physical task parameters (by modifying force levels or employing different muscle groups). Finally, sufficient recovery should be provided post work.

\section{Conclusion}

While a number of studies have documented the influence of cognitive demands on acute physiological responses, few have assessed their effects on muscle fatigue. Findings from the current study provide evidence of the adverse effects of mental demands on muscle capacity. It is therefore important to consider potential changes in worker capacity with concurrent physical and cognitive demands before designing work tasks/products. Applications of these results can aide in the development of ergonomics tools that will incorporate concurrent assessment of physical and cognitive demands in the workplace.

\section{References}

[1] Bernard BP. Musculoskeletal disorders and workplace factors a critical review of epidemiologic evidence for work-related musculoskeletal disorders of the neck, upper extremity, and low back. US Department of Health and Human Services CDC (NIOSH). 1997; Publication No. 97-141.

[2] Birch L, Juul-Kristensen B, Jensen C, Finsen L, Christensen H. Acute response to precision, time pressure and mental demand during simulated computer work. Scandinavian Journal of Work, Environment \& Health. 2000;26(4):299.

[3] Carter JR, Kupiers NT, Ray CA. Neurovascular responses to mental stress. The Journal of Physiology. 2005;564(1):321

[4] Chaudhuri A, Behan PO. Fatigue and basal ganglia. Journal of the neurological sciences. 2000;179(1-2):34-42.

[5] Hjortskov N, Rissen D, Blangsted AK, Fallentin N, Lundberg $\mathrm{U}$, Søgaard K. The effect of mental stress on heart rate varia- bility and blood pressure during computer work. European Journal of Applied Physiology. 2004;92:84-9.

[6] Holding D. Fatigue. In: Hockey GRJ, editor. Stress and fatigue in human performance. New York: John Wiley and Sons Ltd.; 1983. p. 145-67.

[7] Laursen B, Jensen BR, Garde AH, Jorgensen AH. Effect of mental and physical demands on muscular activity during the use of a computer mouse and a keyboard. Scandinavian Journal of Work Environment and Health. 2002;28:215-21.

[8] Lorist M, Kernell D, Meijman T, Zijdewind I. Motor fatigue and cognitive task performance in humans. The Journal of Physiology. 2002;545(1):313.

[9] Lundberg U. Psychophysiology of work: Stress, gender, endocrine response, and work-related upper extremities disorders. American Journal of Industrial Medicine. 2002;41:383-92.

[10]Lundberg U, Forsman M, Zachau G, Ekloèf M, Palmerud G, Melin B, et al. Effects of experimentally induced mental and physical stress on motor unit recruitment in the trapezius muscle. Work \& Stress. 2002;16(2):166-78.

[11]Lundberg U, Kadefors R, Melin B, Palmerud G, Hassmen P Engstrom M, et al. Psychophysiological Stress and EMG Activity of the Trapezius Muscle. International Journal of Behavioral Medicine. 1994;1(4):354-70.

[12] Macdonell CW, Keir PJ. Interfering effects of the task demands of grip force and mental processing on isometric shoulder strength and muscle activity. Ergonomics. 2005;48(15):1749-69.

[13] Mehta RK, Agnew MJ. Effects of concurrent physical and mental demands for a short duration static task. International Journal of Industrial Ergonomics. 2011;41(5):488-93.

[14]NRC. Musculoskeletal disorders and the workplace: Low back and upper extremities. 2001.

[15] Schleifer LM, Spalding TW, Kerick SE, Cram JR, Ley R, Hatfield BD. Mental stress and trapezius muscle activation under psychomotor challenge: A focus on EMG gaps during computer work. Psychophysiology. 2008;45(3):356 - 65.

[16] van Loon EM, Masters RSW, Ring C, McIntyre DB. Changes in limb stiffness under conditions of mental stress. Journal of Motor Behavior. 2001;33(2):153-64.

[17] Veiersted KB. Sustained muscle tension as a risk factor for trapezius myalgia. International Journal of Industrial Ergonomics. 1994;14(4):333-9.

[18] Vøllestad NK. Measurement of human muscle fatigue. Journal of Neuroscience Methods. 1997;74:219-27.

[19] Waersted M, Bjorklund RA, Westgaard RH. Shoulder muscle tension induced by two VDU-based tasks of different complexity. Ergonomics. 1991;34(2):137-50.

[20] Welford AT. Stress and performance. Ergonomics. 1973;16(5):567-80.

[21] Yoon T, Keller ML, De-Lap BS, Harkins A, Lepers R, Hunter SK. Sex differences in response to cognitive stress during a fatiguing contraction. Journal of Applied Physiology. 2009;107(5):1486. 